\title{
ÍNDICE DA QUALIDADE DO SONO DE PITTSBURGH PARA USO NA REABILITAÇÃO CARDIOPULMONAR E METABÓLICA
}

Original Article ARTIGo Original Artículo Original

\author{
PITTSBURGH SLEEP QUALITY INDEX FOR USE IN CARDIOPULMONARY \\ AND METABOLIC REHABILITATION \\ ÍNDICE DE CALIDAD DEL SUEÑO DE PITTSBURGH PARA USO EN \\ LA REHABILITACIÓN CARDIOPULMONAR Y METABÓLICA
}

Pablo Antonio Bertasso de Araujo ${ }^{1}$ (Educador Físico)

Sabrina Weiss Sties ${ }^{1,2}$

(Fisioterapeuta)

Priscilla Geraldine Wittkopf ${ }^{1}$

(Fisioterapeuta)

Almir Schmitt Netto

(Educador Físico)

Ana Inês Gonzáles ${ }^{1}$ (Fisioterapeuta)

Daiane Pereira Lima

(Fisioterapeuta)

Samantha Nahas Guimarães

(Educadora Física)

Eduardo Eugenio Aranha

(Educador Físico)

Alexandro Andrade 4

(Educador Físico)

Tales De Carvalho

(Médico Cardiologista)

1. Universidade do Estado de Santa Catarina (UDESC) ,Centro de Ciências da Saúde e do Esporte (CEFID), Núcleo de Cardiologia e Medicina do Exercício (NCME), Florianópolis, SC, Brasil.

2. Faculdade Avantis, Balneário

Camboriú, SC, Brasil.

3. Fundação Catarinense de Educação Especial (FCEE), São José, SC, Brasil.

4. Universidade do Estado de Santa Catarina (UDESC), Centro de Ciências da Saúde e do Esporte (CEFID), Laboratório de Psicologia do Esporte e do Exercício (LAPE), Florianópolis, SC, Brasil.

\section{Correspondência:}

Centro de Ciências da Saúde e do Esporte (CEFID/UDESC), Núcleo de Cardiologia e Medicina do Exercício. Rua Pascoal Simone, 358, bloco C. Coqueiros, Florianópolis, SC, Brasil. 88080-350

sabrinasties@yahoo.com.br

\section{RESUMO}

Introdução: A qualidade do sono constitui-se em parâmetro relevante na avaliação da saúde em geral, sendo um fator relevante na determinação do risco das doenças cardiovasculares. Objetivo: Validar a versão adaptada do questionário de avaliação do Índice de Qualidade do Sono de Pittsburgh (PSQI) para uso em programas de reabilitação cardiopulmonar e metabólica (RCPM). Métodos: Estudo descritivo transversal realizado com 101 pacientes de ambos os sexos, com média de idade de 66,05 $( \pm 13,9)$ anos. Para a análise estatística foi considerado intervalo de confiança de $95 \%$ e valor de significância $\mathrm{p}<0,05$; para a análise de consistência interna foi utilizado o coeficiente de alfa de Cronbach e para a análise da relação entre componentes e itens com o escore total do questionário foi utilizado o coeficiente de correlação de Spearman. Resultados: Todos os componentes do questionário apresentaram boa consistência interna com valor de 0,72. Os componentes que mais se relacionaram com o escore total foram "duração do sono" e "qualidade subjetiva do sono", sendo que o componente que menos se relacionou foi "alterações do sono". Dentre os itens a variação foi de 0,584 no item "durante a última semana, em geral, como você classificaria a qualidade do seu sono?", até -0,611 no item "durante a última semana, quantas horas você conseguia dormir durante a noite?". Foi possível observar que os itens "tossir ou roncar muito alto" e "frequência para dificuldades do sono por outras razões" não apresentaram correlação com o escore total do questionário. Conclusão: A versão adaptada do PSQI mostrou-se válida para ser utilizada na avaliação do sono em programas de RCPM.

Palavras-chave: reabilitação, doenças cardiovasculares, transtornos do sono.

\section{ABSTRACT}

Introduction: Sleep quality is a relevant parameter in the assessment of overall health, and a relevant factor in determining the risk of cardiovascular diseases. Objective: To validate the adapted version of the Pittsburgh Sleep Quality Index (PSQI) for use in cardiopulmonary and metabolic rehabilitation (CPMR) programs. Methods: Descriptive cross-sectional study conducted with 101 patients of both sexes, average age of $66.05( \pm 13.9)$ years. For statistical analysis, it was considered a confidence interval of $95 \%$ and significance value of $p<0.05$; for internal consistency analysis the Cronbach's alpha was used and for analysis of the relationship among components and items and the total score of the questionnaire the Spearman correlation was used. Results: All components of the questionnaire showed good internal consistency with a value of 0.72. The components that most closely related to the total score were "duration of sleep" and "subjective quality of sleep", and the least related was "sleep changes". Among the items, the variation was 0.584 in item "during the last week, in general, how would you rate the quality of your sleep?", until -0.611 in item "during the last week, how many hours could you sleep at night?". We could observe that the items "cough or snore too high" and "frequency of sleep difficulties for other reasons" were not correlated with the total score of the questionnaire. Conclusion: The adapted PSQI version is valid for use in sleep assessment in CPMR programs.

Keywords: rehabilitation, cardiovascular diseases, sleep disorders.

\section{RESUMEN}

Introducción: La calidad del sueño constituye un parámetro relevante en la evaluación de la salud en general, y es un factor importante para determinar el riesgo de enfermedades cardiovasculares. Objetivo: Validar la versión adaptada del cuestionario de evaluación del Índice de Calidad del Sueño de Pittsburgh (PSQI) para su uso en programas de rehabilitación cardiopulmonar y metabólica (RCPM). Métodos: Estudio descriptivo de corte transversal que se llevó a cabo con 101 pacientes de ambos sexos, con edad promedio de 66,05 (土13,9) años. Para el análisis estadístico, un intervalo de confianza del $95 \%$ y valor de significación de $p<0,05$ fueran considerados; para el análisis de consistencia interna se utilizó el alfa de Cronbach y para el análisis de la relación entre los componentes y 
elementos con la puntuación total del cuestionario fue utilizado el el coeficiente de correlación de Spearman. Resultados: Todos los componentes del cuestionario mostraron una buena consistencia interna, con un valor de 0,72. Los componentes más estrechamente relacionados con la puntuación total fueron "duración del sueño" y "calidad subjetiva del sueño", y el componente menos relacionado fue "cambios en el sueño". Entre los elementos, la variación fue de 0,584 en "¿durante la última semana, en general, cómo usted calificaría la calidad de su sueño?", hasta -0,611 en "idurante la última semana, cuántas horas se podía dormir por la noche?". Se observó que los elementos "tos o ronquido demasiado alto" y "frecuencia de dificultades para dormir por otras razones" no se correlacionaron con la puntuación total del cuestionario. Conclusión: La versión adaptada del PSQI ha demostrado ser válida para su uso en la evaluación del sueño en programas del RCPM.

Palabras clave: rehabilitación, enfermedades cardiovasculares, trastornos del sueño.

\section{INTRODUÇÃO}

A má qualidade do sono, que apresenta prevalência de oito a dezoito por cento na população em geral ${ }^{1}$ e de 50 a 70\% na população de idosos ${ }^{2}$, está fortemente associada às doenças cardiovasculares $(\mathrm{DCV})^{3,4}$ e a mortalidade total ${ }^{5-7}$.

Estudos sugerem que a má qualidade do sono constitui fator de risco para agravamento das DCV, podendo também ser um importante marcador da saúde cardiovascular ${ }^{4}$. Há comprovada relação entre a má qualidade e a duração do sono com uma série de fatores de risco independentes para a doença arterial coronariana $(\mathrm{DAC})^{8}$, como a hipertensão arterial sistêmica ${ }^{9}$, o diabetes mellitus ${ }^{10}$ e a obesidade ${ }^{11}$.

A qualidade do sono tem papel relevante no imprescindível tratamento das DCV, como a DAC e a insuficiência cardíaca (IC), por meio da reabilitação cardiopulmonar e metabólica (RCPM) que corresponde a modificações de estilo de vida favoráveis à saúde, com ênfase na prática de exercício físico $(E F)^{12}$. Para avaliar a qualidade da intervenção, tem sido demonstrado que o EF regular quando corretamente executado contribui para melhorar a qualidade do sono ${ }^{13,14}$. No entanto, quando mal executado o EF pode prejudicar a qualidade do sono, como ocorre na síndrome do excesso de treinamento ${ }^{15}$.

Portanto, avaliar a qualidade do sono pode contribuir para o tratamento das DCV no contexto da RCPM, possibilitando ajustes terapêuticos relacionados à prescrição de EF, por exemplo. O exame de polissonografia é considerado o padrão ouro para avaliação do sono. No entanto, é limitado quanto à disponibilidade e custo econômico em relação à demanda, situações que dificultam sua viabilidade e adoção na saúde pública.

Assim, podem ser considerados instrumentos como o Índice da Qualidade do Sono de Pittsburg (PSQI) ${ }^{16}$, pois tem demonstrado ser eficaz e capaz de fornecer informações quantitativas e qualitativas acerca da qualidade do sono ${ }^{16-18}$. No entanto, sempre que um novo questionário é criado ou traduzido, ele deve ser validado, para a nova população na qual será utilizado ${ }^{19}$. Diante do exposto o objetivo do presente estudo foi validar a versão adaptada do questionário de avaliação do Índice de Qualidade do Sono de Pittsburg (PSQI) para uso em programas de reabilitação cardiopulmonar e metabólica (RCPM).

\section{MÉTODO}

Trata-se de um estudo descritivo de corte transversal com amostragem não probabilística.

Fizeram parte do estudo 101 indivíduos de ambos os sexos, participantes de um programa de RCPM, sendo 52,5\% homens e 47,5\% mulheres, com média de idade de 66.05 ( \pm 9.13$)$ anos. A tabela 1 apresenta as características dos participantes do estudo.
Tabela 1. Características dos participantes.

\begin{tabular}{|c|c|}
\hline Características & $\mathrm{N}(\%)$ \\
\hline \multicolumn{2}{|c|}{ Classificação socioeconômica } \\
\hline Classe A & $8(8)$ \\
\hline Classe B & $61(61)$ \\
\hline Classe C & $30(30)$ \\
\hline Classe D e E & $1(1)$ \\
\hline \multicolumn{2}{|l|}{ Estado nutricional } \\
\hline Baixo Peso & $9(9,5)$ \\
\hline Eutrófico & $31(32,6)$ \\
\hline Sobrepeso & $20(21,1)$ \\
\hline Obesidade & $35(36,8)$ \\
\hline \multicolumn{2}{|l|}{ Diagnósticos } \\
\hline DAC & $62(62,6)$ \\
\hline IC & $5(5,1)$ \\
\hline HAS & $65(33,7)$ \\
\hline Diabetes & $31(31,3)$ \\
\hline Dislipidemia & $17(16,8)$ \\
\hline DAOP & $11(11,1)$ \\
\hline
\end{tabular}

N: amostra; \%:percentual; DAC: doença arterial coronariana; IC: Insuficiência cardíaca; HAS: hipertensão arterial sistêmica; DAOP: doença arterial obstrutiva periférica.N: amostra; \%:percentual; DAC: doença arterial coronariana; IC: Insuficiência cardíaca; HAS: hipertensão arterial sistêmica; DAOP: doença arterial obstrutiva periférica.

Inicialmente foi utilizado um questionário semiestruturado com perguntas que abordaram aspectos referentes ao diagnóstico médico e fatores de risco cardiovasculares (hipertensão/diabetes/dislipidemia). Para classificação socioeconômica foi utilizado o Critério Padrão de Classificação Econômica Brasil/2008 da Associação Brasileira das Empresas de Pesquisa ${ }^{20}$. Esse questionário avalia itens existentes na residência do participante e a escolaridade do chefe da família. O questionário tem alta relação com a renda familiar $\left(r=0,78\right.$ e $\left.r^{2}=62 \%\right)$.

$O$ estado nutricional foi verificado por meio do índice de massa corporal (IMC), o qual foi obtido pela razão entre massa corporal e estatura ao quadrado $\left(\mathrm{Kg} / \mathrm{m}^{2}\right)^{21}$. Para a classificação do estado nutricional de indivíduos até 60 anos de idade, adotaram-se os valores de referência propostos pela Organização Mundial da Saúde 22 e para pacientes acima de 60 anos os valores de referência adotados foram os propostos pela Organização Pan-Americana de Saúde ${ }^{23}$.

\section{Questionário Índice de Qualidade do Sono de Pittsburgh (PSQI)}

O PSQI avalia a qualidade e perturbações do sono durante o período de um mês e foi desenvolvido por Buysse et al. ${ }^{16}$, sendo um questionário padronizado, simples e bem aceito pelos pacientes ${ }^{19}$.

O instrumento é constituído por 19 questões em auto-relato e cinco questões direcionadas ao cônjuge ou acompanhante de quarto. As últimas cinco questões são utilizadas apenas para a prática clinica, não contribuindo para a pontuação total do índice. As 19 questões 
são categorizadas em sete componentes, graduados em escores de zero (nenhuma dificuldade) a três (dificuldade grave). Os componentes do PSQI são: C1 qualidade subjetiva do sono, C2 latência do sono, C3 duração do sono, C4 eficiência habitual do sono, C5 alterações do sono, C6 uso de medicamentos para dormir C7 disfunção diurna do sono. A soma dos valores atribuídos aos sete componentes varia de zero a vinte e um no escore total do questionário indicando que quanto maior o número pior é a qualidade do sono. Um escore total maior que cinco indica que o indivíduo está apresentando grandes disfunções em pelo menos dois componentes, ou disfunção moderada em pelo menos três componentes ${ }^{16,24,25}$.

No Brasil, inicialmente Konrad ${ }^{25}$ realizou a tradução e validação do PSQI (forma curta) em indivíduos com síndrome da fibromialgia. Nessa versão o instrumento sofreu uma adaptação quanto ao tempo de recordação, sendo este tempo reduzido de um mês para a recordação referente à última semana.

Posteriormente, foi realizada tradução e validação do PSQI-BR em indivíduos com suspeita clínica de apneia obstrutiva do sono ou insônia e depressão ${ }^{24}$. No entanto, até o presente momento, não foram realizados procedimentos para a validação do mesmo em pacientes com doenças cardiopulmonares e metabólicas, participantes de programas de RCPM.

Após explanação dos objetivos do estudo, todos os participantes assinaram o Termo de Consentimento Livre e Esclarecido, aprovado pelo Comitê de Ética em Pesquisa (parecer 149/2011), de acordo com a resolução 196/96 do Conselho Nacional de Saúde. Os pesquisadores agendaram um horário que melhor se adequasse à rotina dos participantes. A coleta de dados foi realizada evitando interferências externas, sendo cada participante do estudo avaliado individualmente por pesquisadores que atuam no programa de RCPM. O questionário foi administrado por pesquisador treinado e o tempo de aplicação foi de 5 a 10 min.

\section{Análise Estatística}

A análise estatística foi realizada no programa Statistical Package for the Social Science - SPSS ${ }^{\circledR}$ versão 20.0 para Windows ${ }^{\circledR}$ considerando intervalo de confiança de 95\% e valor de significância $p<0.05$. Variáveis descritivas foram expressas em média, desvio padrão, mediana e distribuição de frequência. Foi utilizado o alfa de Cronbach para análise de consistência interna. Para análise da relação entre os componentes e itens com o escore total do questionário foi utilizada a correlação de Spearman.

\section{RESULTADOS}

O escore total do PSQI apresentou variação entre zero e dezessete, com média de 5.37 ( \pm 3.64 ) e mediana de quatro. Todos os sete componentes apresentaram variação entre zero e três. No escore total do questionário, 38,6\% dos participantes apresentaram pontuação acima de cinco, indicando disfunção moderada a grave em dois ou três componentes do questionário.

A idade correlacionou-se negativamente com o componente "disfunções diurnas do sono" ( $r=0.205 p<0.041)$. O escore total e os demais seis componentes não apresentaram correlação com a idade.

Os sete componentes apresentaram boa consistência interna avaliada por meio do alfa de Cronbach com valor de 0.72, indicando que cada um dos sete componentes mede um aspecto particular do construto geral.

Os componentes que mais se relacionaram com o escore total (tabela 2) foram "duração do sono" e "qualidade subjetiva do sono", e o que menos se relacionou foi "alterações do sono". A variação foi de 0.584 no item "durante a última semana, em geral, como você classificaria a qualidade do seu sono?", até -0.611 no item "durante a última semana, quantas horas você conseguia dormir durante a noite?". Foi possível observar que os itens "tossir ou roncar muito alto" e "frequência para dificuldades do sono por outras razões" não apresentaram correlação com o escore total do questionário.

Tabela 2. Relação entre os componentes e itens com o escore total do Índice de Qualidade do Sono de Pittsburg.

\begin{tabular}{|c|c|c|}
\hline Componente & Escore total & \\
\hline & r & $p$ \\
\hline Qualidade subjetiva do sono & 0,664 & 0,001 \\
\hline Latência do sono & 0,570 & 0,001 \\
\hline Duração do sono & 0,693 & 0,001 \\
\hline Eficiência habitual do sono & 0,601 & 0,001 \\
\hline Alterações do sono & 0,453 & 0,001 \\
\hline Medicações para o sono & 0,491 & 0,001 \\
\hline Disfunção diurna do sono & 0,529 & 0,001 \\
\hline Item 2 & 0,328 & 0,001 \\
\hline Item 4 & $-0,611$ & 0,001 \\
\hline Item $5 \mathrm{a}$ & 0,470 & 0,001 \\
\hline Item 5b & 0,324 & 0,001 \\
\hline Item $5 c$ & 0,205 & 0,040 \\
\hline Item 5d & 0,326 & 0,023 \\
\hline Item 5e & 0,145 & 0,149 \\
\hline Item $5 f$ & 0,316 & 0,001 \\
\hline Item $5 \mathrm{~g}$ & 0,245 & 0,014 \\
\hline Item 5h & 0,405 & 0,001 \\
\hline Item $5 \mathrm{i}$ & 0,287 & 0,004 \\
\hline Item $5 j$ & 0,115 & 0,252 \\
\hline Item 6 & 0,471 & 0,001 \\
\hline Item 7 & 0,308 & 0,002 \\
\hline Item 8 & 0,468 & 0,001 \\
\hline Item 9 & 0,584 & 0,001 \\
\hline
\end{tabular}

r: valor da correlação; p:nível de significância de 5\%.

\section{DISCUSSÃO}

Utilizando a nota de corte de cinco, conforme recomendado por Buysse et al. ${ }^{16}$ no estudo original, 38,6\% dos participantes do presente estudo apresentaram pontuação acima de cinco, sendo classificados como "maus dormidores". Dois estudos brasileiros que utilizaram o PSQI encontraram resultados superiores, em pacientes com insuficiência cardíaca relataram que 68,5\% apresentaram escores maiores ou iguais a cinco, sendo classificados como maus dormidores ${ }^{26}$ e em diabéticos observaram que $52 \%$ dos pacientes apresentaram qualidade do sono ruim ${ }^{27}$. O resultado encontrado em nosso estudo pode ser explicado pelo fato de que os pacientes avaliados participam de um programa de RCPM regularmente, estando o EF associado a uma melhor qualidade de sono ${ }^{14}$.

Com relação à idade, tem sido demonstrado que o envelhecimento está associado à mudança tanto subjetiva quanto objetiva na qualidade do sono ${ }^{5}$, e há uma correlação positiva entre a idade e prevalência de queixas no sono ${ }^{6}$. A idade correlacionou-se negativamente com o componente "disfunções diurnas do sono", no entanto, a literatura aponta que no idoso, o sono torna-se mais superficial e fragmentado e, portanto, de menor eficiência e pior qualidade 28

Refletindo a boa consistência interna, o valor de alfa de Cronbach mostrou que há homogeneidade entre as questões, correspondendo ao valor de 0,72, sendo considerados valores positivos quando acima de 0,6. Nos estudos de validação de Buysse et al. ${ }^{16}$ no artigo original ( $a=0.83$ ), e no estudo de Bertolazi et al..$^{24}$ de validação para o português ( $a=0.82$ ) os valores observados de alfa também mostraram-se acima de 0,6, indi- 
cando um elevado grau de consistência interna. Analisando o valor de alfa de Cronbach, cada um dos sete componentes do PSQI mede um aspecto particular da mesma construção geral, a qualidade do sono ${ }^{16}$.

Os componentes que mais se relacionaram com o escore total foram "duração do sono" e "qualidade subjetiva do sono", e o que menos se relacionou foi "alterações do sono", sendo que esses dados foram igualmente observados no artigo original de Buysse et al. ${ }^{16}$. O coeficiente de correlação baixo observado no item "alterações do sono" pode ser o resultado do grande número de itens que compõem este componente, assim como pelo fato de que esses itens podem ser particularmente sensíveis à variação entre os indivíduos e ao tempo ${ }^{16}$.

Foram encontradas variações de 0,584 no item "durante a última semana, em geral, como você classificaria a qualidade do seu sono", até - 0.611 no item "durante a última semana, quantas horas você conseguia dormir durante a noite?". Foi possível observar que o item "tossir ou roncar muito alto" e "frequência para dificuldades do sono por outras razões" não apresentaram correlação com o escore total do questionário, sendo que na questão "tossir ou roncar muito alto" também não foi observada correlação no artigo original16

Contudo, o processo de validação do Índice de Qualidade do Sono de Pittsburg possibilitou o preenchimento de uma lacuna ao qualificar o PSQI para uso em pacientes com doenças cardiopulmonares e metabólicas.

\section{CONCLUSÃO}

A versão adaptada do PSQI mostrou-se válida para ser utilizada em pacientes com doenças cardiopulmonares e metabólicas participantes de RCPM.

Todos os autores declararam não haver qualquer potencial conflito de interesses referente a este artigo.

\section{REFERÊNCIAS}

1. Ohayon MM. Epidemiology of insomnia: what we know and what we still need to learn. Sleep Med Rev. 2002;6(2):97-111.

2. Reid KJ, Baron KG, Lu B, Naylor E, Wolfe L, Zee PC. Aerobic exercise improves self-reported sleep and quality of life in older adults with insomnia. Sleep Med. 2010;11(9):934-40.

3. Bagai K. Obstructive sleep apnea, stroke, and cardiovascular diseases. Neurologist. 2010;16(6):329-39.

4. Sabanayagam C, Shankar A. Sleep duration and cardiovascular disease: results from the National Health Interview Survey. Sleep. 2010;33(8):1037-42.

5. Grandner MA, Hale L, Moore M, Patel NP. Mortality associated with short sleep duration: the evidence, the possible mechanisms, and the future. Sleep Med Rev. 2010;14(3):191-203.

6. Kojima M, Wakai K, Kawamura T, Tamakoshi A, Aoki R, Lin Y, et al. Sleep patterns and total mortality: a 12-year follow-up study in Japan. J Epidemiol. 2000;10(2):87-93.

7. Tamakoshi A, Ohno Y, JACC Study Group. Self-reported sleep duration as a predictor of all-cause mortality: results from the JACC study, Japan. Sleep. 2004;27(1):51-4.

8. Greenland P, Knoll MD, Stamler J, Neaton JD, Dyer AR, Garside DB, et al. Major risk factors as antecedents of fatal and nonfatal coronary heart disease events. JAMA. 2003;290(7):891-7.

9. Vgontzas AN, Liao D, Bixler EO, Chrousos GP, Vela-Bueno A. Insomnia with objective short sleep duration is associated with a high risk for hypertension. Sleep. 2009;32(4):491-7.

10. Spiegel K, Knutson K, Leproult R, Tasali E, Van Cauter E. Sleep loss: a novel risk factor for insulin resistance and Type 2 diabetes. J Appl Physiol (1985). 2005;99(5):2008-19.

11. Watanabe M, Kikuchi H, Tanaka K, Takahashi M. Association of short sleep duration with weight gain and obesity at 1-year follow-up: a large-scale prospective study. Sleep. 2010;33(2):161-7.

12. Cohen-Mansfield J, Perach R. Sleep duration, nap habits, and mortality in older persons. Sleep. 2012;35(7):1003-9.

13. Yang PY, Ho KH, Chen HC, Chien MY. Exercise training improves sleep quality in middle-aged and older adults with sleep problems: a systematic review. J Physiother. 2012;58(3):157-63.

14. Youngstedt SD, Kline CE. Epidemiology of exercise and sleep. Sleep Biol Rhythms. 2006;4(3):215-221.

15. Sties SW, Gonzales Al, Netto AS, Wittkoft PG, Lima DP, Carvalho T. Validação da escala de humor de Brunel para programa de reabilitação cardiovascular. Rev Bras Med Esporte. 2014;20(4):281-4.
16. Buysse DJ, Reynolds CF 3rd, Monk TH, Berman SR, Kupfer DJ. The Pittsburgh Sleep Quality Index: a new instrument for psychiatric practice and research. Psychiatry Res. 1989;28(2):193-213.

17. Buysse DJ, Hall ML, Strollo PJ, Kamarck TW, Owens J, Lee L, et al. Relationships between the Pittsburgh Sleep Quality Index (PSQI), Epworth Sleepiness Scale (ESS), and clinical/polysomnographic measures in a community sample. J Clin Sleep Med. 2008;4(6):563-71.

18. Cole JC, Motivala SJ, Buysse DJ, Oxman MN, Levin MJ, Irwin MR. Validation of a 3-factor scoring mode for the Pittsburgh sleep quality index in older adults. Sleep. 2006;29(1):112-6.

19. Lomeli HA, Pérez-Olmos I, Talero-Gutiérrez C, Moreno CB, González-Reyes R, Palacios L, et al. Sleep evaluation scales and questionaries: a review. Actas Esp Psiquiatr. 2008;36(1):50-9.

20. Associação Brasileira de Empresas de Pesquisa (ABEP). Caracterização socioeconômica populacional: 2012. Disponível em: http://www.abep.org/novo/Content.aspx?ContentID=301. [Acesso em: 06/ jan/2014].

21. Tritschler K (editor). Medida e avaliação em educação física e esportes. São Paulo: Manole; 2003.

22. World Health Organization (WHO). The problem of overweight and obesity. Geneva: WHO; 2000.

23. Organização Pan-americana de la Salud, División de Promoción y Protección de la Salud (HPP). Encuesta multicentrica salud bienestar y envejecimiento (SABE) en América Latina: informe preliminar / Multicenter survey aging, health and wellbeing in Latin América and the Caribbean (SABE): preliminary report. Washington, DC: Organización Panamericana de la Salud; 2001.

24. Bertolazi AN, Fagondes SC, Hoff LS, Dartora EG, Miozzo IC, de Barba ME, et al. Validation of the Brazilian Portuguese version of the Pittsburgh Sleep Quality Index. Sleep Med. 201;12(1):70-5.

25. Konrad LM. Efeito agudo do exercício físico sobre a qualidade de vida de mulheres com síndrome da fibromialgia [dissertação]. Florianópolis: Universidade Federal de Santa Catarina; 2005.

26. Santos MA, Guedes ES, Barbosa RL, Cruz DAL. Dificuldades do sono relatadas por pacientes com insuficiência cardíaca. Rev Latino-Am Enfermagem. 2012;20(4):9-18.

27. Cunha MCB, Zanetti ML, Hass VJ. Qualidade do sono em diabéticos do tipo 2. Rev Latino-Am Enfermagem. 2008;16(5):1-7.

28. Missildine K, Bergstrom N, Meininger J, Richards K, Foreman MD. Sleep in hospitalized elders: a pilot study. Geriatr Nurs. 2010;31(4):263-71. 